Article

\title{
A New UPLC-MS/MS Method for the Characterization and Discrimination of Polysaccharides from Genus Ephedra Based on Enzymatic Digestions
}

\author{
Yong-Gang Xia, Tian-Long Wang, Li-Ming Sun, Jun Liang, Bing-You Yang and Hai-Xue Kuang * \\ Key Laboratory of Chinese Materia Medica, Heilongjiang University of Chinese Medicine, \\ Ministry of Education, Harbin 150040, China; yonggangxia@163.com (Y.-G.X.); wt1890322@163.com (T.-L.W.); \\ llimingsun@163.com (L.-M.S.); lliangjunn@163.com (J.L.); ybywater@163.com (B.-Y.Y.) \\ * Correspondence: hxkuang56@163.com; Tel.: +86-451-8276-7188
}

Received: 29 October 2017; Accepted: 15 November 2017; Published: 17 November 2017

\begin{abstract}
Ephedra sinica polysaccharides have been reported to possess important activities, so quality evaluation of polysaccharides from the genus Ephedra is urgent. In this study, enzymatic digestions were performed to establish multiple saccharide fingerprints by ultra-performance liquid chromatography with electrospray ionization triple quadrupole linear ion trap mass spectrometry (UPLC-ESI-TQ-MS/MS) based on a multiple-reaction monitoring in negative mode. Under optimum UPLC-ESI-TQ-MS/MS conditions, excellent separation and quantification of 21 constituents were achieved within $20 \mathrm{~min}$ on a solid core column with a $1.6 \mu \mathrm{m}$ particle using pre-column derivatization with a PMP reagent. This method, coupled with enzymatic digestions and principal component analysis, has been successfully applied to characterize and discriminate Ephedra polysaccharides attributed to different species and plant parts. The results suggest that the proposed analytical strategy could achieve a quality evaluation of plant polysaccharides from traditional Chinese medicines.
\end{abstract}

Keywords: UPLC-MS/MS; polysaccharides; genus Ephedra; enzymatic digestion

\section{Introduction}

Mahuang is a famous traditional Chinese medicine (TCM) that has been used for thousands of years for the treatment of allergies, asthma, pneumonia, bronchitis, hay fever, and colds [1]. According to the 2015 edition of the Chinese pharmacopoeia, Mahuang is dry stems of Ephedra sinica Stapf, E. intermedia Schrenket C. A. Mey. and E. equisetina Bge., while Radix Ephedrae is recorded to be another different TCM, which is the dry roots or rhizoma of E. sinica and E. intermedia in the official pharmacopoeia of China [2]. Recently, some reports have demonstrated that the stems of E. sinica contain a high amount of polysaccharides, ranging from $3 \%$ to $5 \%$ of the total weight $[3,4]$. In addition, E. sinica stem polysaccharides showed obviously immunosuppressive effects by a carbon clearance test, delayed type hypersensitivity reaction, and humoral immune response in vivo [4-6]. However, so far there is no published information on discrimination of Ephedra polysaccharides from different species and plant parts. Therefore, it is essential to develop an effective method for quality evaluation of Ephedra polysaccharides.

A variety of chromatographic techniques such as gas chromatography (GC) [7], capillary electrophoresis (CE) [8], high-performance liquid chromatography (HPLC) $[9,10]$, and high-performance anion exchange chromatography (HPAEC) $[11,12]$ were previously considered as useful tools for the determination of carbohydrates. Although existing techniques are increasingly mature and gradually improving, they still face many problems and challenges. The limit of quantification of the GC, HPLC-UV, and CE-UV method is too restrictive and cannot be used in trace quantification of 
carbohydrates. HPAEC can achieve more satisfactory separation than HPLC techniques, but the epimerization and degradation can be issues at high $\mathrm{pH}$ [11,12]. Except for HPAEC, most of the LC and CE techniques often use labeling with either fluorescence or UV tags for enhanced detection. The reagent 1-phenyl-3-methyl-5-pyrzolone (PMP) has been widely used for composition analysis of plant polysaccharides [3].

Carbohydrates encompass a number of homologues with similar structures, so the analysis of carbohydrates inevitably requires high-resolution separation techniques. With the progress of chromatographic techniques, ultra-performance liquid chromatography (UPLC) was developed with a sub-2 $\mu \mathrm{m}$ diameter, which shortened the analysis time and provided high resolution and sensitivity separation, especially tandem mass spectrometry (MS/MS). Although UPLC-MS/MS have been applied to analyze plant polysaccharides, most reports are limited to several monosaccharides $[13,14]$.

In this study, UPLC with triple quadrupole linear ion trap mass spectrometry (TQ-MS/MS) was firstly developed for simultaneous analysis of 21 carbohydrates and one degradation product (5-HMF, 5-hydroxymethyl furfural) based on a pre-column PMP derivatization. Elution of all eight neutral sugars, two uronic acids, three amino sugars, and two acetyl amino sugars, was then used to evaluate the performance of this method. Finally, UPLC-ESI ${ }^{-}$-TQ-MS/MS coupled with enzymatic digestions and principal component analysis (PCA) has been successfully applied to analyze and characterize 20 Ephedra polysaccharide samples attributed to different species and plant parts. The results showed that the proposed analytical methods provided reasonable insight and guidance for quality evaluation of plant polysaccharides from the genus Ephedra.

\section{Experimental}

\subsection{Materials and Reagents}

E. sinica, E. intermedia, and E. equisetina were purchased in September 2012 from Datong, Shanxi Province, China and identified by Professor Zhen-Yue Wang of Heilongjiang University of Chinese Medicine. The voucher specimens (20120905) were deposited at the herbarium of Heilongjiang University of Chinese Medicine, Harbin, China.

D-galacturonic acid (GalUA), D-glucuronic acid (GlcUA), D-fucose (Fuc), L-rhamnose (Rha), D-glucose (Glc), D-galactose (Gal), D-mannose (Man), L-arabinose (Ara),D-ribose (Rib), N-Acetyl-D-(+)glucosamine (GlcNAc), N-Acetyl-D-(+)-galactosam-ine (GalNAc), D-glucosamine hydrochloride (GlcN), D-galactosamine hydrochloride (GalN), D-mannosamine hydrochloride (ManN), 5-hydroxymethyl furfural (5-HMF), lactose, maltotriose, maltotetraose, maltopentaose, maltohexaose, and maltoheptaose were purchased from Sigma- Aldrich (St. Louis, MO, USA). D2-Glc, 1-phenyl-3-methyl-5-pyrazolone (PMP), ammonium formate (AF), and formic acid (FA) were also obtained from Sigma-Aldrich. Pectinase, $\alpha$-amylase, and cellulose were purchased from Sigma-Aldrich; xylanase and endo-1,3- $\beta$-glucanase were obtained from Megazyme. Water was obtained from a Milli-Q purification system (Millipore, Bedford, MA, USA). All other chemicals were of the highest analytical grade.

\subsection{Preparation of Polysaccharides from Genus Ephedra}

The dried sample materials (100 g) were extracted three times with 10-fold volume of distilled water under reflux for $3 \mathrm{~h}$ each time. The filtrate of the obtained extract was condensed under a vacuum to syrup and precipitated with $95 \%$ ethanol added to a final concentration of $80 \%$. After standing for $24 \mathrm{~h}$ at $4{ }^{\circ} \mathrm{C}$, the precipitate was collected and washed with anhydrous ethanol, then dried. The residue was re-dissolved in water, and then, after centrifugation (4000 rpm for $15 \mathrm{~min}$ ), the supernatant was dialyzed (cut-off Mw $3500 \mathrm{Da}$ ) against tap water for $48 \mathrm{~h}$ and distilled water for $48 \mathrm{~h}$ and lyophilized to yield crude polysaccharides. The crude polysaccharides, which were prepared in duplicate, were obtained for further analysis. 


\subsection{Specific Enzymatic Digestion of Ephedra Polysaccharides}

Polysaccharide solutions $(5 \mathrm{mg} / \mathrm{mL}, 200 \mu \mathrm{L})$ were mixed with specific enzymes (the final concentrations of pectinase, endo-1,4- $\beta$-xylanase, cellulose, $\beta-(1 \rightarrow 3)$-D-glucanase and $\alpha$-amylase were $30 \mathrm{U} / \mathrm{mL}, 10 \mathrm{U} / \mathrm{mL}, 0.3 \mathrm{U} / \mathrm{mL}, 0.2 \mathrm{U} / \mathrm{mL}$ and $30 \mathrm{U} / \mathrm{mL}$, respectively) in a $1.5-\mathrm{mL}$ centrifuge tube and digested overnight $(\geq 15 \mathrm{~h})$ at $50{ }^{\circ} \mathrm{C}$. Then the mixtures were heated at $80^{\circ} \mathrm{C}$ for $20 \mathrm{~min}$ to stop the enzymatic digestion. After centrifugation $(12,000 \mathrm{rpm} / \mathrm{min})$ at room temperature for $20 \mathrm{~min}$, the supernatants were evaporated to dryness with a nitrogen evaporator at $70{ }^{\circ} \mathrm{C}$ and then used for derivatization with PMP. Moreover, the blank control was treated as described above without enzymes.

\subsection{Derivatization with PMP Reagent}

PMP derivatization of carbohydrates was carried out as described previously with proper modifications [3]. Briefly, PMP was prepared in methanol at a final concentration of $0.5 \mathrm{M}$. One hundred microliters of each individual standard monosaccharide and oligosaccharide, mix standard solutions, and the hydrolyzed polysaccharide samples were placed in 1.5-mL centrifuge tubes. Then, $0.5 \mathrm{M}$ PMP $(100 \mu \mathrm{L})$ and ammonia $(200 \mu \mathrm{L})$ were added. $\mathrm{D}_{2}$-Glc as an internal standard $(2 \mathrm{M})$ was added to each sample $(2 \mu \mathrm{L})$. Each mixture was allowed to react for $20 \mathrm{~min}$ in a $70^{\circ} \mathrm{C}$ water bath, then cooled to room temperature and neutralized with $200 \mu \mathrm{L}$ of formic acid. The solution was directly filtered through a $0.22-\mu \mathrm{m}$ membrane and diluted with deionized water before UPLC-TQ-MS/MS analysis.

\subsection{UPLC Apparatus and Conditions}

An ACQUITY ultra-performance liquid chromatographic system (Waters, Milford, MA, USA) was applied. The chromatographic separation was conducted on a cortecs UPLC $\mathrm{C}_{18}$ column $(1.6 \mu \mathrm{m}, 2.1 \times 100 \mathrm{~mm})$ equipped with a cortecs UPLC $C_{18}$ guard column $(1.6 \mu \mathrm{m}, 2.1 \times 5 \mathrm{~mm})$. The binary mobile phase was composed of acetonitrile as solvent A and deionized water with $10 \mathrm{mM}$ $\mathrm{AF}$ as solvent $\mathrm{B}$. Optimal run time for separation was $20 \mathrm{~min}$ using the following gradient program at a flow rate of $0.3 \mathrm{~mL} / \mathrm{min}$ and a column oven temperature of $30{ }^{\circ} \mathrm{C}$ : isocratic $13 \% \mathrm{~A}$ at $0-0.5 \mathrm{~min}$; $0.5-2 \mathrm{~min}$, linear from $13 \%$ to $16 \% \mathrm{~A}(v / v) ; 2-15 \mathrm{~min}$, linear from $16 \%$ to $21 \% \mathrm{~A}(v / v) ; 15-16 \mathrm{~min}$, linear from $21 \%$ to $60 \%$ A $(v / v) ; 16-17 \mathrm{~min}$, linear from $60 \%$ to $90 \%$ A $(v / v)$; followed by column re-equilibration using $13 \% \mathrm{~A}$ at $17-20 \mathrm{~min}$. The injection volume was set to $2 \mu \mathrm{L}$.

\subsection{MS Apparatus and Conditions}

The MS detection was performed using an API 4000 Qtrap (AB Sciex, Foster City, CA, USA) equipped with an electrospray ionization (ESI) interface, and operated in negative ion mode with multiple reaction monitoring (MRM). Turbo $\mathrm{V}$ ion source parameters were common to all analytes, as follows: the capillary voltage was $-4.5 \mathrm{kV}$, and the source temperature was at $550{ }^{\circ} \mathrm{C}$. The curtain gas and collision gas setting were $30 \mathrm{psi}$ and $10 \mathrm{psi}$, respectively. The pressure for nebulization gas and vaporization gas setting were 55 psi. The declustering potential (DP) and collision energy (CE) were optimized for each analyte.

\subsection{Data Processing}

Evaluation of the different polysaccharides from genus Ephedra could be facilitated using multivariate statistical analysis. PCA was applied to visualize the data structure, classify the samples according to their components and detect corresponding markers. The PCA was carried out using SPSS version 16.0 (SPSS, Inc., Chicago, IL, USA). 


\section{Results and Discussion}

\subsection{Derivatization and MS Conditions}

In order to achieve a fast and accurate sample preparation, a simplified PMP-derivatization procedure was developed. The PMP reaction mechanism was illustrated in Figure 1A. This modified procedure was characterized by extreme simplification without using any liquid-liquid extraction and concentrated steps by comparison with our previous reports [3]. The resulting solution was directly filtered and diluted with deionized water before UPLC-ESI ${ }^{-}-\mathrm{TQ}-\mathrm{MS} / \mathrm{MS}$. Furthermore, the comparative experiments between before and after liquid-liquid extraction showed that the simplified workflow did not affect the chromatographic separation and MS signal intensities at all. This simplified sample preparation was applied successfully in the current study.
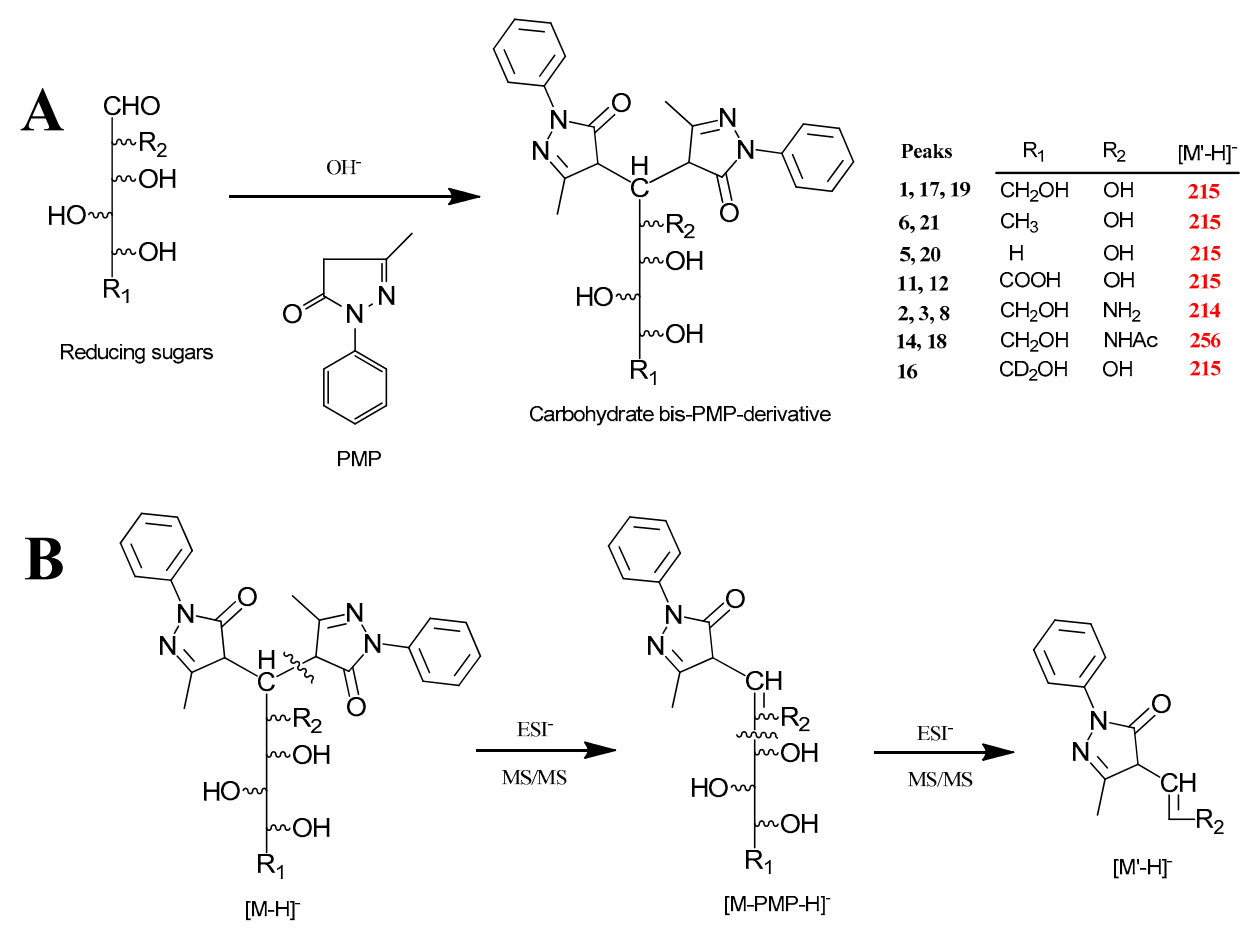

Figure 1. (A) Condensation reaction with PMP; (B) characteristic fragmentation pathways of bis-PMP derivatives of monosaccharides in negative ESI-MS/MS mode. Peaks were as follows: 1, Man; 2, GlcN; 3, ManN; 5, Rib; 6, Rha; 8, GalN; 11, GlcUA; 12, GalUA; 14, GlcNAc; 16, D2-Glc; 17, Glc; 18, GalNAc; 19, Gal; 20, Ara; 21, Fuc.

Regarding MS detection, carbohydrate PMP derivatives could be monitored under both ESI ${ }^{-}$ and $\mathrm{ESI}^{+}$modes. In the present work, much less noise was observed in the $\mathrm{ESI}^{-}$than $\mathrm{ESI}^{+}$modes. Furthermore, the $[\mathrm{M}-\mathrm{H}]^{-}$precursor ions were more intense for all analytes than $[\mathrm{M}+\mathrm{H}]^{+}$and $[\mathrm{M}+\mathrm{Na}]^{+}$, so $[\mathrm{M}-\mathrm{H}]^{-}$precursor ions were used for the detection of the carbohydrate PMP derivatives. Taking collision-induced dissociations of deprotonated PMP-labeled monosaccharides as examples, peaks 1-3, 5, 6, 8, 11, 12, 14, 16 and 17-21 produced similar fragmentation patterns. The typical ESI ${ }^{-}$ fragmentation pathway is illustrated in Figure $1 \mathrm{~B}$. The $[\mathrm{M}-\mathrm{H}]^{-}$precursor first experienced the loss of a PMP unit to afford a $[\mathrm{M}-\mathrm{PMP}-\mathrm{H}]^{-}$ion, which further produced $\alpha$-cleavage to give characteristic $\left[\mathrm{M}^{\prime}-\mathrm{H}\right]^{-}$ions at $m / z 214,215$ or 256 . This $\left[\mathrm{M}^{\prime}-\mathrm{H}\right]^{-}$was considered to be a characteristic product ion based on the fact that this fragment was always stable with prominent intensity when using a specific collision energy. Therefore, the experimental MRM transition used those intact deprotonated $m / z$ values of precursors $\left(Q_{1}\right)$ and characteristic fragments $\left[\mathrm{M}^{\prime}-\mathrm{H}\right]^{-}$as product ions $\left(\mathrm{Q}_{3}\right)$ for accurate detection of bis-PMP derivatives of monosaccharides-3, 5, 6, 8, 11, 12, 14 and 16-21. As a contrast, 
bis-PMP derivatives of oligosaccharides 4, 7, 9, 10, 13 and 15 showed more complex fragmentation mechanism in $\mathrm{ESI}^{-}-\mathrm{MS} / \mathrm{MS}$ mode, so the most intense product ion was chosen as $\mathrm{Q}_{3}$.

Finally, the optimal DP and CE values for all MRM transitions (1-22) are summarized in Table 1. Another advantage of the current method in comparison with traditional HPLC-UV methods is that it could effectively eliminate the interference of the PMP ion peak using UPLC-ESI ${ }^{-}-\mathrm{TQ}-\mathrm{MS} / \mathrm{MS}$ with MRM detection.

Table 1. Mass spectrometry parameters used for analysis of targeted saccharides and internal standards (IS).

\begin{tabular}{ccccccc}
\hline Peaks & Original $\boldsymbol{M}_{\boldsymbol{w}}$ & PMP Derivatives $(\mathbf{m} / \boldsymbol{z})$ & $\mathbf{Q}_{\mathbf{1}}(\mathbf{m} / \boldsymbol{z})$ & $\mathbf{Q}_{\mathbf{3}}(\mathbf{m} / \mathbf{z})$ & $\mathbf{D P}(\mathbf{V})$ & $\mathbf{C E}(\mathbf{V})$ \\
\hline $\mathbf{1}$ & 180.2 & {$[\mathrm{M}-\mathrm{H}]^{-}$} & 509.1 & 215.1 & -49.2 & -28.1 \\
$\mathbf{2}$ & 215.6 & {$[\mathrm{M}-\mathrm{H}]^{-}$} & 508.2 & 214.0 & -65.9 & -29.8 \\
$\mathbf{3}$ & 215.6 & {$[\mathrm{M}-\mathrm{H}]^{-}$} & 508.4 & 213.7 & -64.5 & -26.0 \\
$\mathbf{4}$ & 1153.0 & {$[\mathrm{M}-\mathrm{H}]^{-}$} & 1481.9 & 1308.0 & -96.4 & -35.1 \\
$\mathbf{5}$ & 150.1 & {$[\mathrm{M}-\mathrm{H}]^{-}$} & 478.7 & 215.0 & -58.4 & -29.9 \\
$\mathbf{6}$ & 164.2 & {$[\mathrm{M}-\mathrm{H}]^{-}$} & 493.2 & 214.9 & -60.6 & -29.0 \\
$\mathbf{7}$ & 990.9 & {$[\mathrm{M}-\mathrm{H}]^{-}$} & 1319.7 & 869.8 & -99.5 & -61.2 \\
$\mathbf{8}$ & 215.6 & {$[\mathrm{M}-\mathrm{H}]^{-}$} & 508.2 & 213.8 & -67.9 & -33.2 \\
$\mathbf{9}$ & 828.7 & {$[\mathrm{M}-\mathrm{H}]^{-}$} & 1157.7 & 707.6 & -90.3 & -51.7 \\
$\mathbf{1 0}$ & 666.6 & {$[\mathrm{M}-\mathrm{H}]^{-}$} & 995.6 & 214.8 & -80.8 & -52.6 \\
$\mathbf{1 1}$ & 212.2 & {$[\mathrm{M}-\mathrm{H}]^{-}$} & 523.2 & 215.0 & -55.9 & -30.3 \\
$\mathbf{1 2}$ & 194.1 & {$[\mathrm{M}-\mathrm{H}]^{-}$} & 523.1 & 215.0 & -57.5 & -47.4 \\
$\mathbf{1 3}$ & 504.4 & {$[\mathrm{M}-\mathrm{H}]^{-}$} & 833.6 & 215.3 & -98.4 & -46.8 \\
$\mathbf{1 4}$ & 221.2 & {$[\mathrm{M}-\mathrm{H}]^{-}$} & 550.2 & 256.2 & -63.9 & -32.9 \\
$\mathbf{1 5}$ & 360.3 & {$[\mathrm{M}-\mathrm{H}]^{-}$} & 671.5 & 214.8 & -61.2 & -34.1 \\
$\mathbf{1 6}$ & 182.2 & {$[\mathrm{M}-\mathrm{H}]^{-}$} & 511.2 & 214.6 & -63.7 & -28.0 \\
$\mathbf{1 7}$ & 180.2 & {$[\mathrm{M}-\mathrm{H}]^{-}$} & 509.0 & 214.7 & -59.9 & -26.1 \\
$\mathbf{1 8}$ & 221.2 & {$[\mathrm{M}-\mathrm{H}]^{-}$} & 550.3 & 256.0 & -69.4 & -34.1 \\
$\mathbf{1 9}$ & 180.2 & {$[\mathrm{M}-\mathrm{H}]^{-}$} & 509.2 & 215.0 & -65.0 & -29.9 \\
$\mathbf{2 0}$ & 150.1 & {$[\mathrm{M}-\mathrm{H}]^{-}$} & 479.0 & 215.1 & -48.4 & -33.9 \\
$\mathbf{2 0}$ & 150.1 & {$[\mathrm{M}-\mathrm{H}]^{-}$} & 479.1 & 215.0 & -63.1 & -28.1 \\
$\mathbf{2 1}$ & 164.2 & {$[\mathrm{M}-\mathrm{H}]^{-}$} & 493.1 & 215.1 & -54.5 & -30.4 \\
$\mathbf{2 2}$ & 126.1 & {$[\mathrm{M}-\mathrm{H}]^{-}$} & 455.4 & 173.1 & -60.9 & -14.1 \\
\hline
\end{tabular}

Peaks 1-3, 5, 6, 8, 11, 12, 15 and 16-21 were the same as in Figure 1. Other peaks are as follows: 4, maltoheptaose; 7, maltohexaose; 9, maltopentaose; 10, maltotetraose; 13, maltotriose; 15, lactose; 22, 5-HMF.

\subsection{Quantification and Validation}

The chromatographic conditions were optimized to achieve the best resolution of various saccharides of interest within a short analysis. Some carbohydrate PMP derivatives might have different structures, but have the same precursor ion and daughter ion. So it is difficult to fully separate them by common HPLC techniques. Thus, UPLC-ESI ${ }^{-}$-TQ-MS/MS was explored to separate and detect carbohydrates. To establish an ideal separation, the chromatographic parameters involving column types, additives of mobile phases, flow rates and column temperatures were optimized.

\subsubsection{Effects of Columns}

The UPLC method was developed to achieve a possible separation of various carbohydrate PMP derivatives of interest. The result showed that some tested PMP derivatives were inadequately separated $(\mathbf{2}$ and $\mathbf{3}, \mathbf{5}$ and $\mathbf{6})$ by BEH C $18(2.1 \times 100 \mathrm{~mm}, 1.7 \mu \mathrm{m})$ or available at a lower MS response intensity $(\mathbf{1}, \mathbf{2}, \mathbf{3}, \mathbf{8}, \mathbf{1 1}, \mathbf{1 2}$ and 16-19) using the HSS T3 column $(2.1 \mathrm{~mm} \times 100 \mathrm{~mm}, 1.8 \mu \mathrm{m})$ and ACE $\mathrm{C}_{18}(150 \times 2.1 \mathrm{~mm}, 3 \mu \mathrm{m})$ or a longer retention time up to 20-30 $\mathrm{min}(\mathbf{1 6}-19)$ employing a HSS T3 column $(2.1 \mathrm{~mm} \times 150 \mathrm{~mm}, 1.8 \mu \mathrm{m})$ and BEH C $18(2.1 \times 100 \mathrm{~mm}, 1.7 \mu \mathrm{m})$. Therefore, a cortecs UPLC $\mathrm{C} 18(2.1 \times 100 \mathrm{~mm}, 1.6 \mu \mathrm{m})$ column was used in this work to detect PMP derivatives 1-22. It was found that this specific column could provide high column efficiency and good MS response while retaining a relatively short run time. 


\subsubsection{Effects of Additives}

To obtain chromatograms with good resolution and high intensity, various additives such as acetic acid, FA, and AF were investigated in the pre-experiment, and AF was shown to give the best separation and peak shape. The effects of additive AF concentrations (8 mM, $10 \mathrm{mM}, 15 \mathrm{mM}$ and $10 \mathrm{mM}$ AF with $0.1 \%$ FA) were also investigated on resolutions and MS response intensities. The results showed the moderate $10 \mathrm{mM}$ AF showed the highest MS intensity for most of the peaks tested. If $10 \mathrm{mM}$ AF with a lower $\mathrm{pH}$ was applied, it seemed to make some peaks co-eluted, such as 1-3 and 5-8. Therefore, $10 \mathrm{mM}$ AF was used for further, optimized steps.

\subsubsection{Effects of Column Temperature and Flow Rate}

The effects of different column temperatures $\left(25^{\circ} \mathrm{C}, 30^{\circ} \mathrm{C}\right.$ and $\left.35^{\circ} \mathrm{C}\right)$ were assayed. Generally, all carbohydrate PMP derivatives tested were eluted to be a sharp peak at column temperatures of $25-35{ }^{\circ} \mathrm{C}$; there was no obvious effect on the peak symmetry and resolution of eluted analytes. However, peak responses were much better when the column temperature was applied at $30{ }^{\circ} \mathrm{C}$. Therefore, the column temperature of $30^{\circ} \mathrm{C}$ was used for further optimization steps. The effect of different flow rates $(0.2,0.3$ and $0.4 \mathrm{~mL} / \mathrm{min})$ has also been evaluated. After comprehensive consideration of peak resolutions, symmetries, retention time, and column pressures, $0.3 \mathrm{~mL} / \mathrm{min}$ was selected as the optimum flow rate.

Under the above optimum conditions, the MRM chromatograms of reference standards 1-22 are given in Figure 2, which shows the complete baseline separation of all corresponding isomeric carbohydrate PMP derivatives, including 1, 17 and $19\left(\mathrm{Q}_{1} / \mathrm{Q}_{3}, 509 / 215\right), 2$, 3, and $18\left(\mathrm{Q}_{1} / \mathrm{Q}_{3}\right.$, 508/214), 5 and $20\left(\mathrm{Q}_{1} / \mathrm{Q}_{3}, 479 / 215\right), 6$ and $21\left(\mathrm{Q}_{1} / \mathrm{Q}_{3}, 493 / 215\right)$, and 11 and $12\left(\mathrm{Q}_{1} / \mathrm{Q}_{3}, 523 / 215\right)$. These targeted analytes include 14 monosaccharides (1-3, 5, 6, 8, 11, 12, 14, 17-21), one internal standard compound (16), six oligosaccharides $(4,7,9,10,13$ and 15) and one degraded product (22). Although some peaks such as 1, 2, 5, 6, 10-14 and 16-18 were not fully separated in total ion chromatography (Supplementary materials Figure S1), they were still accurately quantified due to the presence of different MRM selections. It is worth mentioning that this is the first example on simultaneous analysis of eight neutral sugars $(\mathbf{1}, \mathbf{5}, \mathbf{6}, \mathbf{1 6}, \mathbf{1 7}, \mathbf{1 9 - 2 1 )}$, two uronic acids (11 and 12), three amino sugars (2, 3 and 8$)$, two acetyl amino sugars (14 and 17), six oligosaccharides $(4,7,9,10$, 13 and 15), and one degradation product (22) using UPLC-ESI ${ }^{-}-\mathrm{TQ}-\mathrm{MS} / \mathrm{MS}$.

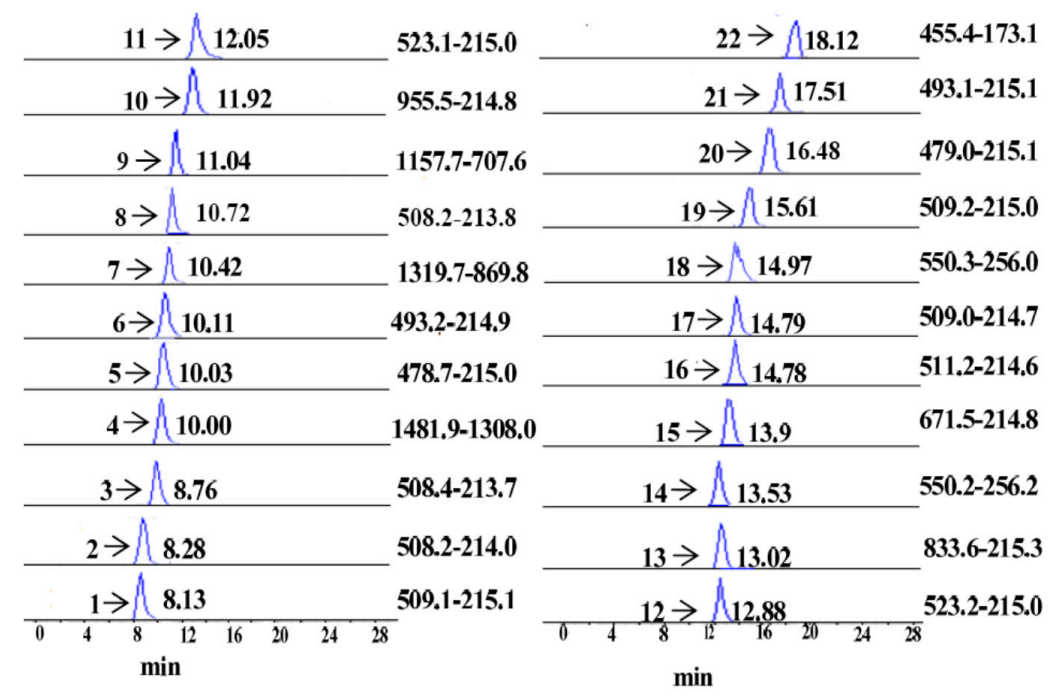

Figure 2. MRM ion chromatograms and 22 reference standards. Peaks 1-3, 5, 6, 8, 11, 12, 15 and 16-21 are the same as in Figure 1. Other peaks are as follows: 4, maltoheptaose; 7, maltohexaose; 9, maltopentaose; 10, maltotetraose; 13, maltotriose; 15, lactose; 22, 5-HMF. 


\subsection{Method Validations}

The analytical procedure has been validated in order to confirm its reliability. Table 2 summarizes the calibration curves, LOD and LOQ values of the carbohydrate PMP derivatives analyzed by UPLC-ESI ${ }^{-}$- TQ-MS/MS with MRM mode. Since it is difficult to be precise about sample preparations due to the presence of derivatization procedures, the internal standard method is used. Here synthetic $D_{2}$-Glc was chosen to be the internal standard in terms of different $Q_{1} / Q_{3} M R M$ selection. All the peaks showed good linearity $\left(R^{2}>0.99\right)$ in quite a wide concentration range. The LOD and LOQ were determined at a signal-to-noise (S/N) of about 3 and 10, respectively. The results showed that the LODs of monosaccharides and oligosaccharides were in the range of 3-48 nM and 12-780 nM, respectively, indicating that the sensitivity of the method was satisfactory. The intra-day and inter-day RSD values of the analytes were all less than $4.86 \%$, implying good precision and reproducibility. For stability testing, the same sample solution was analyzed with 21 analytes every $4 \mathrm{~h}$ over $24 \mathrm{~h}$ with RSD less than $4.03 \%$, which indicated that the sample was stable over two days under experimental conditions.

Table 2. Summarization of calibration results, LOD, and LOQ values.

\begin{tabular}{cccccc}
\hline No. & Calibration Curves & $\boldsymbol{R}^{\mathbf{2}}$ & Linear Range $(\boldsymbol{\mu M})$ & LOD $(\mathbf{n M})$ & LOQ $(\mathbf{n M})$ \\
\hline $\mathbf{1}$ & $y=0.2634 x+0.1916$ & 0.9938 & $0.02-49.60$ & 3.00 & 8.00 \\
$\mathbf{2}$ & $y=0.1721 x+0.0360$ & 0.9982 & $0.19-49.60$ & 24.00 & 58.00 \\
$\mathbf{3}$ & $y=0.2135 x+0.2631$ & 0.9931 & $0.05-49.60$ & 12.00 & 34.00 \\
$\mathbf{4}$ & $y=0.0002 x+0.0002$ & 0.9995 & $3.10-49.60$ & 780.00 & 2240.00 \\
$\mathbf{5}$ & $y=0.4846 x+0.2466$ & 0.9960 & $0.02-49.60$ & 3.00 & 92.00 \\
$\mathbf{6}$ & $y=0.1496 x+0.1560$ & 0.9952 & $0.19-49.60$ & 48.00 & 140.00 \\
$\mathbf{7}$ & $y=0.0012 x+0.0013$ & 0.9938 & $0.78-49.60$ & 193.00 & 640.00 \\
$\mathbf{8}$ & $y=0.1692 x+0.2124$ & 0.9920 & $0.05-49.60$ & 12.00 & 48.00 \\
$\mathbf{9}$ & $y=0.0042 x+0.0009$ & 0.9985 & $0.19-49.60$ & 97.00 & 260.00 \\
$\mathbf{1 0}$ & $y=0.0325 x-0.0110$ & 0.9952 & $0.10-49.60$ & 12.00 & 34.00 \\
$\mathbf{1 1}$ & $y=0.0661 x+0.0919$ & 0.9955 & $0.05-49.60$ & 24.00 & 48.00 \\
$\mathbf{1 2}$ & $y=0.0223 x+0.0342$ & 0.9935 & $0.39-49.60$ & 32.00 & 96.00 \\
$\mathbf{1 3}$ & $y=0.0144 x-0.0040$ & 0.9967 & $0.10-49.60$ & 48.00 & 16.00 \\
$\mathbf{1 4}$ & $y=0.1207 x+0.0956$ & 0.9938 & $0.05-49.60$ & 12.00 & 34.00 \\
$\mathbf{1 5}$ & $y=0.0317 x-0.0026$ & 0.9989 & $0.02-49.60$ & 48.00 & 14.00 \\
$\mathbf{1 7}$ & $y=0.3381 x+0.2201$ & 0.9973 & $0.02-49.60$ & 3.00 & 6.00 \\
$\mathbf{1 8}$ & $y=0.3737 x+0.1974$ & 0.9954 & $0.02-49.60$ & 12.00 & 36.00 \\
$\mathbf{1 9}$ & $y=0.6961 x+0.4415$ & 0.9954 & $0.02-49.60$ & 3.00 & 10.00 \\
$\mathbf{2 0}$ & $y=0.6961 x+0.4415$ & 0.9954 & $0.02-49.60$ & 3.00 & 10.00 \\
$\mathbf{2 1}$ & $y=0.3787 x+0.4894$ & 0.9925 & $0.19-49.60$ & 24.00 & 72.00 \\
$\mathbf{2 2}$ & $y=0.4953 x-0.2220$ & 0.9972 & $0.05-49.60$ & 2.00 & 5.00 \\
\hline
\end{tabular}

Peaks 1-22 were the same as Table 1. Peak 16 was the internal standard compound.

Furthermore, recovery experiments were performed in order to investigate the accuracy of the method. Known amounts of carbohydrate solutes were added to the sample with same procedures including extraction and hydrolysis, and the resulting spiked sample was subjected to the entire analytical sequence. The recovery rate of the method was $94.37 \%-104.37 \%$, with RSD less than $4.23 \%$, suggesting that the method is accurate and practical for the composition analysis in plant polysaccharides.

The occurrence of matrix effects in UPLC-ESI ${ }^{-}$-TQ-MS/MS is well known and has an important impact on the determination of the constituents in complex plant samples. A matrix effect was obtained by relative recoveries: relative recoveries $=($ sample contents after adding - original contents $) /$ contents of standard solutions for adding. The relative recoveries for all compounds ranged between $94.94 \%$ and $100.75 \%$, thus showing the minimal matrix suppression or enhancement of this method. 


\subsection{Application to Real Samples}

After PMP derivatization of Ephedra polysaccharide-treated enzymatic digestions, it was directly injected and separated under the optimum conditions described above. The results of multiple enzymatic digestions are shown in Supplementary materials Table S1. Figure 3A-E shows typical MRM ion chromatograms of E. sinica root polysaccharides using UPLC-ESI ${ }^{-}$-TQ-MS/MS before and after multiple enzymatic digestions, respectively. When the $\alpha$-amylase was used for the treatment of polysaccharides from genus Ephedrae, Ara (20), Glc (17), Gal (19), Man (1), lactose (15), and maltotriose (13) were readily liberated (Figure $3 \mathrm{~A})$. The $\beta-(1 \rightarrow 3)$-D-glucanase digestion could produce both Ara (20) and Glc (17) (Figure 3B), while the pectinase digestion enabled Ara (20), Glc (17), GlcUA (11), GalUA (12), and lactose (15) to be liberated from the core skeletons of Ephedrae polysaccharides (Figure 3C). Meanwhile, a large amount of Glc (17), Ara (20), lactose (15), and maltotriose (13) were freed using the cellulose digestion of Ephedra polysaccharides (Figure 3D). If the endo-1,4- $\beta$-xylanase was applied, the predominant compositional saccharides were detected to be Ara (20), Glc (17), lactose (15), maltotriose (13), and maltotetraose (10) (Figure 3E). Note also that a tetrasaccharide $\left(\mathrm{Glc}^{1}(\alpha) \rightarrow{ }^{4} \mathrm{Glc}(\alpha) \rightarrow{ }^{4} \mathrm{Glc}(\alpha) \rightarrow{ }^{4} \mathrm{Glc}(\alpha)\right)$ residue should be linked in the side chain. The above evidence showed that Ephedra polysaccharides from different species and parts (stems or roots) may possess similar structural characteristics. Therefore, it is difficult to directly classify and discriminate these polysaccharides from genus Ephedra by eye.

A
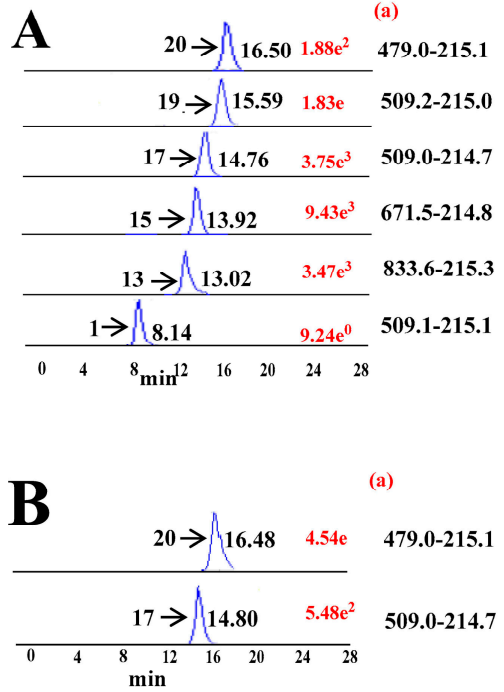

(a)

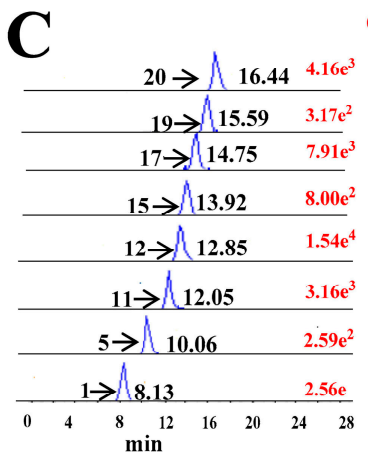

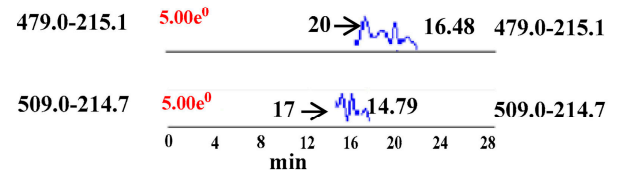

(a)

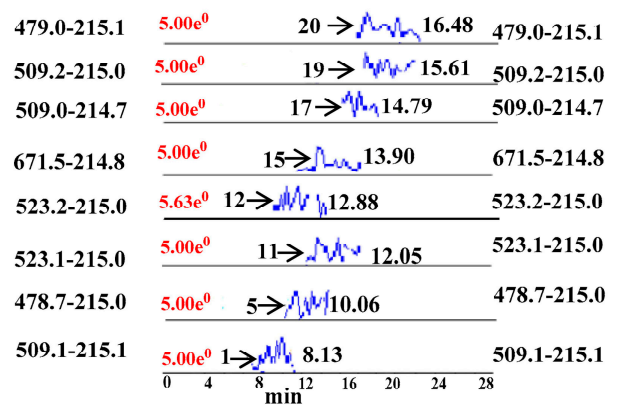

Figure 3. Cont. 


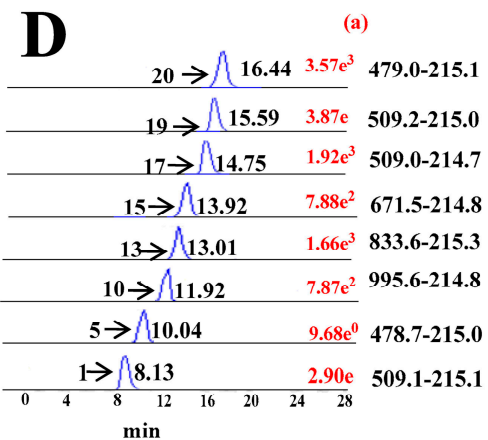

\begin{tabular}{|c|c|c|}
\hline \multirow[b]{2}{*}{$5.00 \mathrm{e}^{0}$} & \multicolumn{2}{|l|}{$20 \rightarrow A+1648$} \\
\hline & $20 \rightarrow M^{16.48}$ & $479.0-215.1$ \\
\hline $5.00 \mathrm{e}^{0}$ & $19 \rightarrow h_{m}-15.61$ & -509.2-215.0 \\
\hline $5.00 \mathrm{e}^{0}$ & $17 \rightarrow p_{14.79}$ & $509.0-214.7$ \\
\hline $5.00 \mathrm{e}^{0}$ & $15 \rightarrow \Omega$ & 671.5-214.8 \\
\hline $5.00 \mathrm{e}^{0}$ & $13 \rightarrow M+13.02$ & $833.6-215.3$ \\
\hline $5.00 \mathrm{c}^{0}$ & $10 \rightarrow x^{\prime} \times 11.92$ & $995.6-214.8$ \\
\hline $5.00 \mathrm{e}^{0}$ & $5 \rightarrow / 4,10.04$ & $-478.7-215.0$ \\
\hline $5.00 \mathrm{e}^{0} 1-$ & 8.13 & 215.1 \\
\hline
\end{tabular}

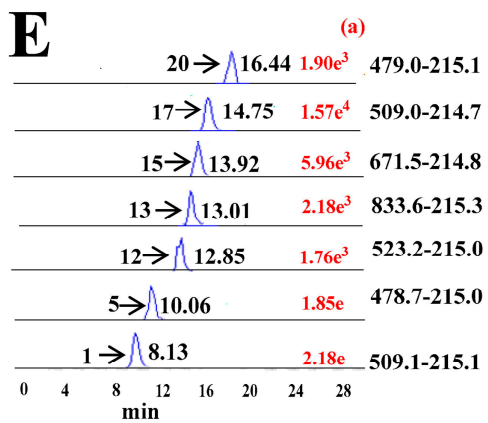

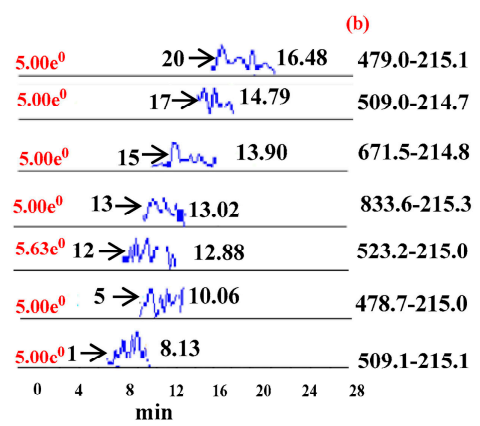

Figure 3. Typical MRM ion chromatograms of E. sinica root polysaccharides treated by multiple enzymatic digestions. (A), $\alpha$-amylase; (B), $\beta-(1 \rightarrow 3)$-D-glucanase, (C), pectinase; (D), cellulose; (E), endo-1,4- $\beta$-xylanase. (a,b) were due to polysaccharide samples with and without enzymatic digestions, respectively.

\subsection{Discrimination of Ephedra Polysaccharides Based on PCA}

As shown in Figure 4A,B,D, 20 Ephedra polysaccharide samples from different species and plant parts were well distinguished using $\alpha$-amylase, $\beta-(1 \rightarrow 3)$-D-glucanase, and cellulose enzymatic digestions, respectively. It was noticeable that samples 1-20 were clustered into four groups, a-d, which were unambiguously identified as polysaccharides from E. sinica stem, E. sinica root, E. intermedia stem, and E. equisetina stem, respectively. The principal components of enzymatic digestions (endo-1,4- $\beta$-xylanase and pectinase) had a minor effect on the model. The typical PCA scores from endo-1,4- $\beta$-xylanase and pectinase digestion are illustrated in Figure 4C,E, respectively.

The PCA loadings (not shown) were used to identify the differential components accountable for the separation among different groups. Ara, Gal, Glc, lactose, Man, and maltotriose were the principal components in classifying these samples for the $\alpha$-amylase digestion of Ephedra polysaccharides. The Ara and Glc may play essential roles in the discrimination of Ephedra polysaccharides treated by the $\beta-(1 \rightarrow 3)$-D-glucanase digestion. Meanwhile, the lactose, maltotriose, Ara, GalUA and Glc could be considered marker components to differentiate these samples using the cellulose digestion. These results further indicated that Ephedra polysaccharides, which were digested by $\alpha$-amylase, $\beta-(1 \rightarrow 3)-D-$ glucanase, and cellulose, produced characteristic saccharide fingerprints. Therefore, UPLC-ESI ${ }^{-}$-TQ-MS/MS coupled with PCA could be used for differentiating Ephedra polysaccharides attributed to different species and plant parts through specific enzymatic digestions. 

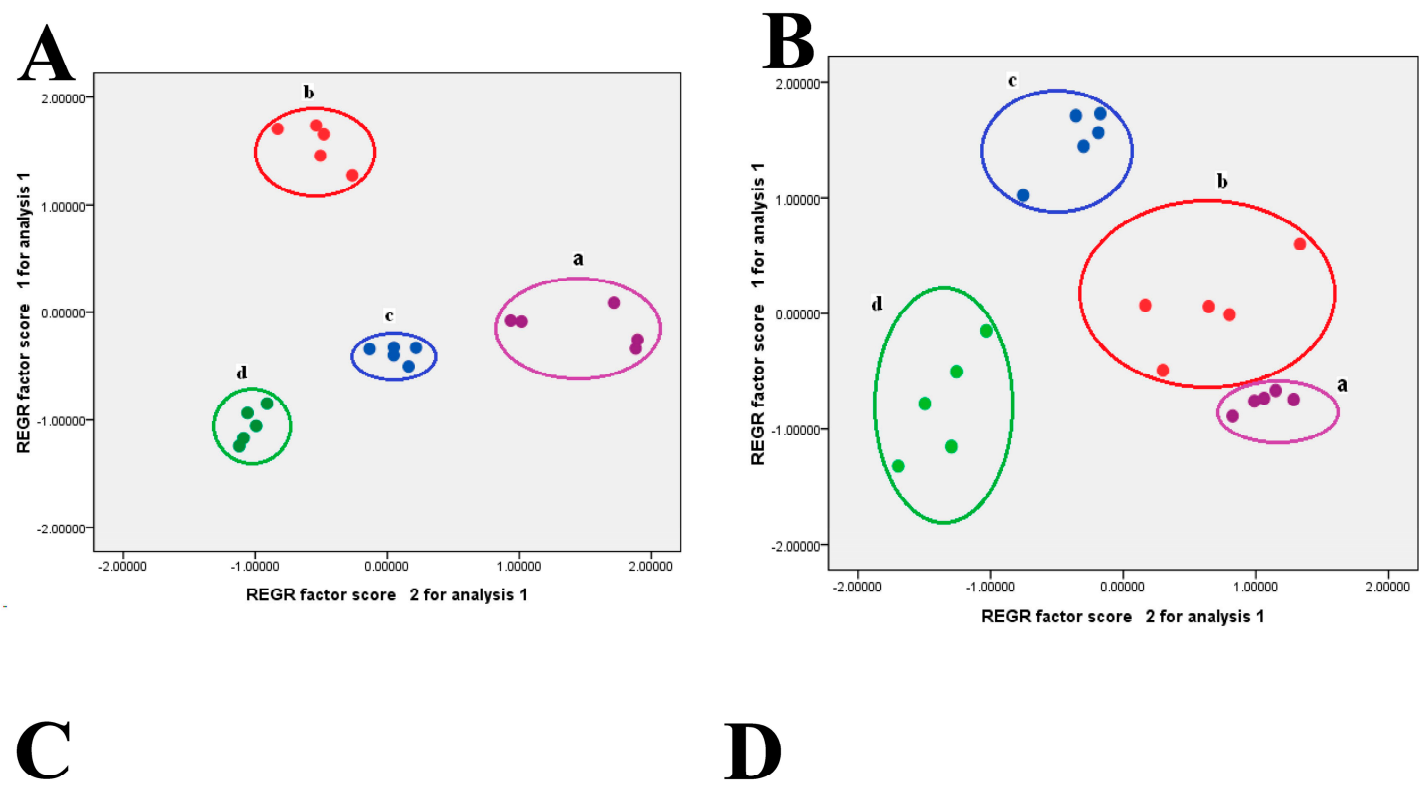

\section{D}
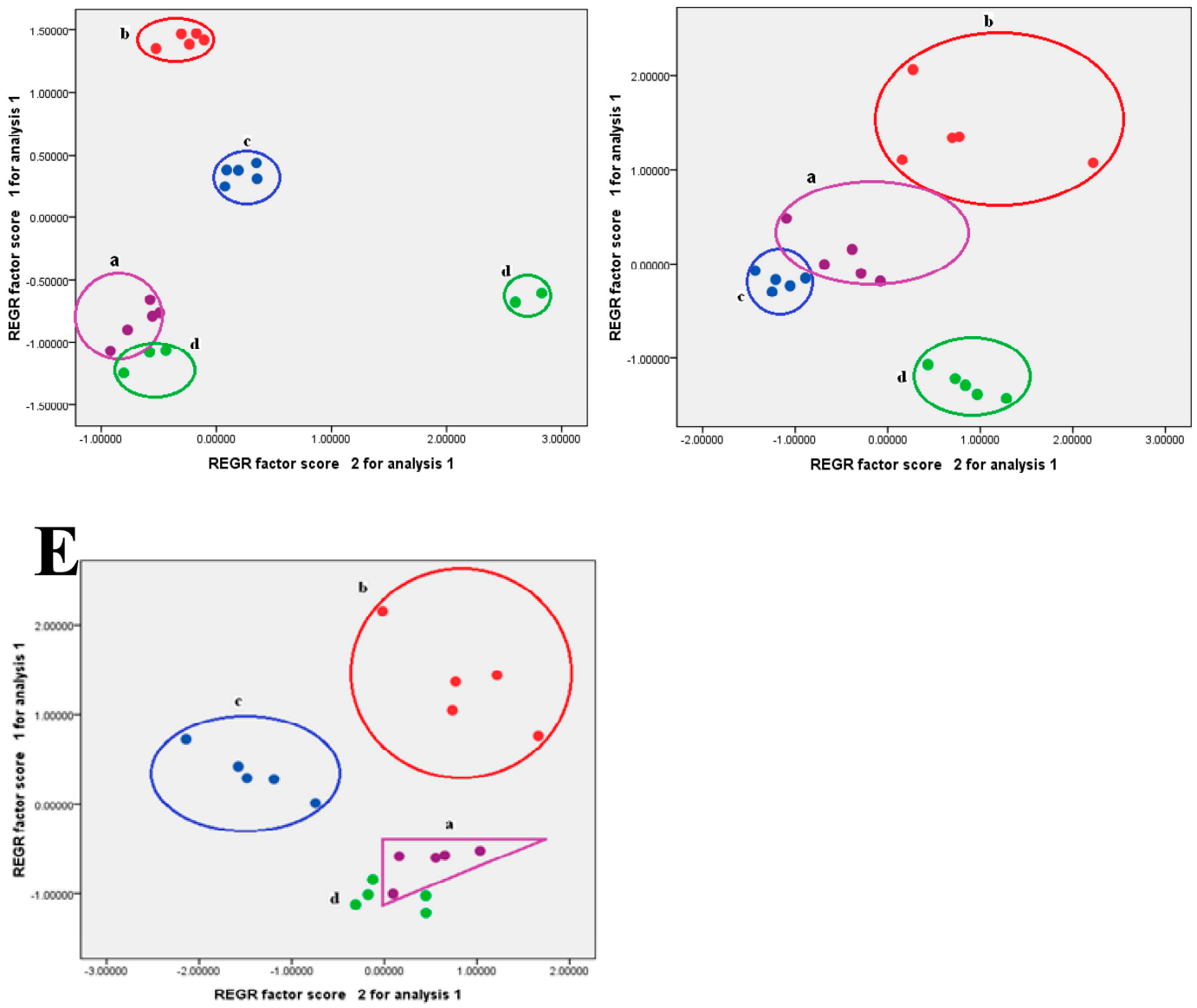

Figure 4. PCA score plots of 20 polysaccharide samples from multiple enzymatic digestions. (A) $\alpha$-amylase; (B) $\beta$-( $1 \rightarrow 3$-D-glucanase, (C) pectinase; (D) cellulose; (E) endo-1,4- $\beta$-xylanase. (a) E. sinica (stem); (b) E. sinica (root); (c) E. intermedia (stem); (d) E. equisetina (stem).

\section{Conclusions}

In this work, a reliable, simple, and sensitive UPLC-ESI ${ }^{-}$-TQ-MS/MS method was developed for the simultaneous analysis of 21 PMP derivatives characterized by the presence of seven neutral 
sugars, two uronic acids, three amino sugars, two acetyl amino sugars, six oligosaccharides, and one degradation product based on a solid core cortecs $C_{18}$ column within $20 \mathrm{~min}$. The proposed method minimizes sample handling, permits high-throughput analysis, and has been successfully applied to analyze 20 Ephedra polysaccharide samples from different species and plant parts. Multivariate statistical analysis results indicated that the specific enzymatic digestions ( $\alpha$-amylase, $\beta-(1 \rightarrow 3)$-D-glucanase, and cellulose) could be used for distinguishing these polysaccharides from genus Ephedra. UPLC-ESI ${ }^{-}$-TQ-MS/MS, coupled with enzymatic digestions and multivariate statistical analysis, may be a powerful and useful approach for quality evaluation of plant polysaccharides from Traditional Chinese Medicine.

Supplementary Materials: Supplementary Materials are available online.

Acknowledgments: Our work was partly financially supported by the National Natural Science Foundation of China (81303215) and the Foundation for the Author of National Excellent Doctoral Dissertation of China (201367).

Author Contributions: H.-X.K., Y.-G.X. and B.-Y.Y. designed this experiment. T.-L.W., L.-M.S. and J.L. participated in data analysis and wrote this paper. All authors read and approved the final manuscript.

Conflicts of Interest: The authors declare no conflict of interest.

\section{References}

1. He, Y.; Zhu, Y.; Zhang, R.; Ge, L.; Wan, H. Simultaneous quantification of nine major active components in traditional Chinese prescription Mahuang decoction and the influence of herbal compatibility on their contents. Pharmacogn. Mag. 2014, 10, 72-79.

2. Pharmacopoeia Commission. Pharmacopoeia of the People's Republic of China 2015; China Medical Science and Technology Press: Beijing, China, 2015; p. 320.

3. Kuang, H.; Xia, Y.; Liang, J.; Yang, B.; Wang, Q.; Sun, Y. Fast classification and compositional analysis of polysaccharides from TCMs by ultra-performance liquid chromatography coupled with multivariate analysis. Carbohydr. Polym. 2011, 84, 1258-1266. [CrossRef]

4. Kuang, H.; Xia, Y.; Yang, B.; Wang, Q.; Wang, Y. Screening and comparison of the immunosuppressive activities of polysaccharides from the stems of Ephedra sinica Stapf. Carbohydr. Polym. 2011, 83, 787-795. [CrossRef]

5. Xia, Y.; Kuang, H.; Yang, B.; Wang, Q.; Liang, J.; Sun, Y.; Wang, Y. Optimum extraction of acidic polysaccharides from the stems of Ephedra sinica Stapf by Box-Behnken statistical design and its anti-complement activity. Carbohydr. Polym. 2011, 84, 282-291. [CrossRef]

6. Kuang, H.; Xia, Y.; Liang, J.; Yang, B. Structural characteristics of a hyperbranched acidic polysaccharide from the stems of Ephedra sinica and its effect on T-cell subsets and their cytokines in DTH mice. Carbohydr. Polym. 2011, 86, 1705-1711. [CrossRef]

7. Guadalupe, Z.; Martínez-Pinilla, O.; Garrido, Á.; Carrillo, J.D.; Ayestarán, B. Quantitative determination of wine polysaccharides by gas chromatography-mass spectrometry (GC-MS) and size exclusion chromatography (SEC). Food Chem. 2012, 131, 367-374. [CrossRef]

8. Hu, Y.; Tong, W.; Yang, X.; Yan, Z. Analysis of compositional monosaccharides in fungus polysaccharides by capillary zone electrophoresis. Carbohydr. Polym. 2014, 102, 481-488. [CrossRef] [PubMed]

9. Muir, J.G.; Rose, R.; Rosella, O.; Liels, K.; Barrett, J.S.; Shepherd, S.J.; Gibson, P.R. Measurement of short-chain carbohydrates in common Australian vegetables and fruits by high-performance liquid chromatography (HPLC). J. Agric. Food Chem. 2009, 57, 554-565. [CrossRef] [PubMed]

10. Zhao, R.B.; Liu, M.J.; Ge, W.; Cui, T.; Liu, W.H. Determination of carbohydrates in Chinese Jujube by using HPLC. Food Sci. 2004, 25, 138-142.

11. Lee, Y.C. High-performance anion-exchange chromatography for carbohydrate analysis. Anal. Biochem. 1990, 189, 151-162. [CrossRef]

12. Garcia, L.M.; Pauli, E.D.; Cristiano, V.; Da, C.C.; Scarminio, I.S.; Nixdorf, S.L. Chemometric evaluation of adulteration profile in coffee due to corn and husk by determining carbohydrates using HPAEC-PAD. J. Chromatogr. Sci. 2009, 47, 825-832. [CrossRef] [PubMed] 
13. Ghfar, A.A.; Wabaidur, S.M.; Ahmed, A.Y.B.H.; Alothman, Z.A.; Khan, M.R.; Al-Shaalan, N.H. Simultaneous determination of monosaccharides and oligosaccharides in dates using liquid chromatography-electrospray ionization mass spectrometry. Food Chem. 2015, 176, 487-492. [CrossRef] [PubMed]

14. Leijdekkers, A.G.M.; Sanders, M.G.; Schols, H.A.; Gruppen, H. Characterizing plant cell wall derived oligosaccharides using hydrophilic interaction chromatography with mass spectrometry detection. J. Chromatogr. 2011, 1218, 9227-9235. [CrossRef] [PubMed]

Sample Availability: Compounds 1-22 are available from the authors.

(C) 2017 by the authors. Licensee MDPI, Basel, Switzerland. This article is an open access article distributed under the terms and conditions of the Creative Commons Attribution (CC BY) license (http://creativecommons.org/licenses/by/4.0/). 